\title{
Acute Bilateral Retinal Arterial Occlusion of Unknown Origin during a Cystic Fibrosis Pulmonary Exacerbation
}

Helton $\mathbf{R}^{*}$ and Allen $\mathrm{K}$

Department of Pulmonology and Critical Care Medicine, University of Oklahoma, Oklahoma City, Oklahoma, USA

\begin{abstract}
Cystic fibrosis is a genetic disorder caused by a defective protein, cystic fibrosis transmembrane conductance regulator. The most common complications of this disease involve the pulmonary, gastrointestinal, and endocrine systems, though there are increasing reports of ocular complications related to cystic fibrosis. Here we report a case of a patient with bilateral retinal artery occlusion of unknown etiology.
\end{abstract}

Keywords: Cystic fibrosis; Branch retinal artery occlusion; Central retinal artery occlusion

\section{Introduction}

Central retinal artery occlusion (CRAO) is a rare event with an incidence of approximately 1 to 10 in 100,000 with symptomatic branch retinal artery occlusion (BRAO) being even less common [1]. CRAO usually present with acute and profound loss of vision that is painless, while BRAO presents with monocular visual loss, which is typically localized to just part of the visual field [2,3]. Carotid artery atherosclerosis is the most common etiology for retinal artery occlusion overall but is rare in younger patients [4]. In younger patients, a cardiogenic source is the most common cause with atrial fibrillation, rheumatic heart disease, or cardiac valvular disease attributed to the underlying cause [4]. Other potential causes described include vasculitis such as giant cell arteritis and hypercoagulable states [5-7]. Currently there is limited literature involving retinal artery occlusion in cystic fibrosis patients, with the only case report found the CRAO related to sinus surgery and triamcinolone injections [8].

\section{Case Report}

A 31-year-old male with cystic fibrosis (CF) presented to our university hospital as a direct floor admission from the adult cystic fibrosis clinic for an acute pulmonary exacerbation. The patient had not routinely followed with a pulmonologist or a primary care physician for more than 5 years. He was non-compliant with his home airway clearance regimen, pancreatic enzymes, and did not take nutritional supplements. He would intermittently receive acute medical care through outside hospital emergency departments with his last emergency department visit a few months prior to current hospitalization. Social history was significant for a history of intravenous heroin abuse but he reported being abstinent for a couple months prior to admission. The patient had never previously been diagnosed with cystic fibrosis related diabetes. Following admission, he was started on intravenous antibiotics (piperacillin/tazobactam, ciprofloxacin, vancomycin) and an airway clearance regimen. Blood cultures on admission were negative and sputum culture grew Staphylococcus aureus and two colonies of Pseudomonas aeruginosa. He was placed on venous thromboembolism prophylaxis with enoxaparin but no assessment of efficacy by factor Xa levels were obtained. His respiratory symptoms improved over the course of the hospitalization.

On day 14 of admission, the patient reported a decrease in vision in the bottom left quadrant of his right eye. He also noted that he developed a hole in his vision of his left eye the previous afternoon. Ophthalmology was consulted and performed an ophthalmologic exam via dilated fundus ophthalmoscopy. The dilated fundus exam of the left eye showed edema along the superior margin of optic nerve with retinal whitening extending along superior vasculature. The right eye exam showed a white horizontal lesion involving superior half of the fovea. These findings and clinical symptoms were compatible with extensive complete branch retinal arterial occlusion in the right eye and a complete cilioretinal arterial occlusion in the left eye. The patient underwent an extensive workup to determine etiology. Repeat blood cultures on the day of the vision loss grew Enterococcus faecium in 1 out of 4 bottles but were later deemed to be a contaminant. Magnetic resonance angiography (MRA) and magnetic resonance imaging (MRI) of the brain were unremarkable. Duplex ultrasound of the bilateral carotid and vertebral arteries were normal with no lesions identified. Bilateral lower extremity duplex ultrasounds showed no evidence of venous thrombosis. A transthoracic echo was unremarkable so cardiology proceeded with a transesophageal echocardiogram (TEE). The TEE showed no thrombus or valvular lesions but was significant for a patent foramen ovale. While on telemetry the patient was always in normal sinus rhythm with no atrial fibrillation or other significant arrhythmia. Rheumatology was consulted and an extensive work up for vasculitis was performed which was negative for ANCA, ANA, anti-myeloperoxidase and anti-proteinase 3 , active hepatitis $\mathrm{B}$ and $\mathrm{C}$, HIV, RPR, and sickle cell. There was no hypergammaglobulinemia and homocysteine levels were normal on subsequent lab work. Hypercoagulability panel (Factor V Leiden, prothrombin gene mutation, protein $\mathrm{C} \& \mathrm{~S}$ deficiency, antithrombin deficiency, antiphospholipid syndrome, fibrinogen, and Russell viper venom mix time) were negative. Electrolytes and renal function were stable and within normal limits during the hospitalization. He was noted to have an increased Westergreen ESR (2x the upper limit of normal). Vascular medicine was later consulted and unable to determine clear etiology of vision loss. The patient was started on anticoagulants and later discharged home. Although he had intermittent follow up, he did not develop any additional retinal artery occlusions while on anticoagulation with persistent vision loss.

*Corresponding author: Richard Helton, Department of Pulmonology and Critical Care Medicine, University of Oklahoma, Oklahoma City, Oklahoma, USA, Tel: 5809270222; E-mail: richard-helton@ouhsc.edu

Received December 10, 2018; Accepted December 20, 2018; Published December 27, 2018

Citation: Helton R, Allen K (2018) Acute Bilateral Retinal Arterial Occlusion of Unknown Origin during a Cystic Fibrosis Pulmonary Exacerbation. J Pulm Respir Med 8: 481. doi: 10.4172/2161-105X.1000481

Copyright: $\odot 2018$ Helton R, et al. This is an open-access article distributed under the terms of the Creative Commons Attribution License, which permits unrestricted use, distribution, and reproduction in any medium, provided the original author and source are credited. 


\section{Discussion}

Central retinal artery occlusion (CRAO) is a rare event with an incidence of approximately 1 to 10 in 100,000 with symptomatic branch retinal artery occlusion (BRAO) being even less common. Currently there is limited literature involving retinal artery occlusion in cystic fibrosis patients, with the only case report found the CRAO related to sinus surgery and triamcinolone injections [9]. In younger patients, a cardiogenic source is the most common cause but in this case there were no risk factors such as atrial fibrillation, rheumatic heart disease, or cardiac valvular disease. The patient did have a history of drug use but with no recent use and no evidence of infective endocarditis, it was determined unlikely to be the cause of the BRAO. There are two reports of retinal vein occlusions in patients with cystic fibrosis. In one case a patient with cystic fibrosis developed bilateral central retinal vein occlusion that was secondary to hyperhomocysteinemia and hypergammaglobulinemia [9], and the other case the patient developed a unilateral branch retinal vein occlusion believed to be secondary to elevated fibrinogen levels [10]. Although the mechanism of pathology in retinal vein and retinal artery occlusion are different, the etiologies may overlap. In our patient, there was no evidence of hypergammaglobulinemia, hyperhomocysteinemia, or elevated fibrinogen levels that would explain the patient retinal artery occlusion. The patient was found to have an elevated ESR which likely reflects the inflammation associated with his pulmonary exacerbation and his underlying cystic fibrosis. Studies have shown that cystic fibrosis pulmonary exacerbations are associated with an elevated ESR and an elevated baseline ESR levels are a predictor of future exacerbations [11]. Another study found that patients with cystic fibrosis had a higher incidence of venous thromboembolism (VTE), and those with higher ESR or CRP had a higher risk to develop a VTE [12]. The patient did have a risk factor for thrombosis given his history of previous intravenous drug use; however, a full work up did not elicit any underlying etiology. In conclusion, the attributed cause of this patient's branch retinal artery occlusion was related to underlying hypercoagulable state resulting from acute on chronic inflammation secondary to his cystic fibrosis pulmonary exacerbation.

\section{Conflict of Interest}

The author(s) declare(s) that there is no conflict of interest regarding the publication of this paper.

\section{References}

1. Leavitt JA, Larson TA, Hodge DO, Gullerud RE (2011) The incidence of central retinal artery occlusion in olmsted county, minnesota. Am J Ophthalmol 152: 820-823.

2. Werner MS, Latchaw R, Baker L, Wirtschafter JD (1994) Relapsing and remitting central retinal artery occlusion. Am J Ophthalmol 118: 393.

3. Yuzurihara D, lijima $\mathrm{H}$ (2004) Visual outcome in central retinal and branch retinal artery occlusion. Jpn J Ophthalmol 48: 490-492.

4. Brown GC, Magargal LE, Shields JA, Goldberg RE, Walsh PN (1981) Retinal arterial obstruction in children and young adults. Ophthalmology 88: 18-25.

5. Mohan K, Gupta A, Jain IS, Banerjee CK (1989) Bilateral central retinal artery occlusion in occult temporal arteritis. J Clin Neuroophthalmol 9: 270-272.

6. Dori D, Beiran I, Gelfand Y, Lanir N, Scharf J, et al. (2000) Multiple retinal arteriolar occlusions associated with coexisting primary antiphospholipid syndrome and factor V Leiden mutation. Am J Ophthalmol 129: 106-108.

7. Dhar-Munshi S, Ayliffe WH, Jayne D (1999) Branch retinal arteriolar occlusion associated with familial factor $V$ Leiden polymorphism and positive rheumatoid factor. Arch Ophthalmol 117: 971-973.

8. Graham DA (2013) Central retinal artery occlusion after frontal sinus triamcinolone injection. EyeNet Magazine.

9. Gelman R, DiMango EA, Schiff WM (2013) Sequential bilateral central retinal vein occlusions in a cystic fibrosis patient with hyperhomocysteinemia and hypergamma-globulinemia. Retin Cases Brief Rep 7: 362-367.

10. Hiscox RJ, Purslow C, North RV, Ketchell I, Evans KS (2014) Branch retina vein occlusion in an asymptomatic adult with cystic fibrosis. Optom Vis Sci 91: S52-S54.

11. Loh G, Ryaboy I, Skabelund A, French A (2018) Procalcitonin, erythrocyte sedimentation rate and C-reactive protein in acute pulmonary exacerbations of cystic fibrosis. Clin Respir J 12: 1545-1549.

12. Knight-Perry J, Branchford BR, Thornhill D, Martiniano SL, Sagel SD, et al (2017) Venous thromboembolism in children with cystic fibrosis: Retrospective incidence and intrapopulation risk factors. Thromb Res 158: 161-166. 